\title{
Schwannoma plexiforme en eminencia tenar
}

\section{Plexiform Schwannoma in Tenar Eminence}

\author{
Miriam Rodríguez Rodríguez ${ }^{1}$ María Ángeles García Frasquet ${ }^{1}$ María Isabel Nieto Díaz de los Bernardos ${ }^{1}$ \\ 1 Servicio de Cirugía Ortopédica y Traumatología del Hospital \\ Universitario Virgen Macarena, Sevilla, Spain \\ Address for correspondence Miriam Rodríguez Rodríguez, Servicio de \\ Cirugía Ortopédica y Traumatología del Hospital Universitario Virgen \\ Macarena, Sevilla, España (e-mail: briank_5@hotmail.com).
}

Rev Iberam Cir Mano 2019;47:39-43.

\section{Resumen \\ Palabras Clave \\ - schwannoma \\ - plexiforme \\ - mano}

El schwannoma plexiforme es un tumor raro, que suele aparecer en tronco, cabeza y cuello. Su diagnóstico definitivo es anatomopatológico, siendo de especial relevancia su distinción con el neurofibroma plexiforme. No se ha descrito potencial de malignización y el tratamiento de elección es la extirpación quirúrgica. Presentamos el caso de un varón de 34 con una tumoración dolorosa en eminencia tenar derecha. Tras realizar RNM que sugiere hemangioma cavernoso, se interviene quirúrgicamente realizándose escisión de la tumoración, cuyo estudio histológico diagnostica de schwannoma plexiforme. A los 12 meses de la cirugía, el paciente se encuentra asíntomático, sin mostrar signos de recidiva o enfermedades asociadas.

Plexiform schwannoma is a rare condition that usually presents in the subcutaneous tissue of trunk, head and neck. The definitive diagnosis is based on anatomopathological findings, being plexiform neurofibroma its main differential diagnosis. Malignant transformation of these tumors has not been previously described and surgical excision is the treatment of choice. A case of a 34-year-old male with a painful mass in the thenar eminence of his right hand is presented. MRI suggested cavernous hemangioma and surgical removal of the tumor was performed. Histological analysis confirmed the diagnosis of plexiform schwannoma. At eleven months follow up, the patient was asymptomatic, showing no signs of recurrence or associated diseases.

\section{Introducción}

El schwannoma plexiforme es una variante inusual del schwannoma descrita por primera vez en 1978 por Harkin y col., que representa menos del $5 \%$ de todos los schwannomas. ${ }^{1}$ Compuesto por la proliferación de células de Schwann de la vaina de los nervios periféricos, se caracteriza por un patrón de crecimiento intraneural y multinodular. Suele presentarse en adultos jóvenes como una masa indolora, de localización subcutánea, consistencia blanda y crecimiento lento. ${ }^{2} \mathrm{~A}$ diferencia del schwannoma convencional que se localiza generalmente en las regiones flexoras de las extremidades, esta variante plexiforme aparece con más frecuencia en cabeza y cuello, seguidos del tronco.

Aunque las técnicas de imagen pueden ser útiles, el diagnóstico definitivo se obtiene mediante el análisis histopatológico, mostrando las características áreas Antoni A..$^{1,2}$

Mientras que los casos esporádicos se presentan como tumores solitarios, la literatura describe una asociación sindrómica con la neurofibromatosis tipo 2 en casos de lesiones múltiples. ${ }^{3}$

Debe diferenciarse especialmente del neurofibroma plexiforme dadas las implicaciones pronósticas de esa received

September 18, 2017

accepted

June 18, 2018
DOI https://doi.org/

10.1055/s-0038-1667128. ISSN 1698-8396.
Copyright $\odot 2019$ Thieme Revinter

Publicações Ltda, Rio de Janeiro, Brazil
License terms

(c) (i) $\ominus$ (\$) 
última entidad, patognomónica de neurofibromatosis tipo potencialmente maligna. $^{3}$

La exéresis quirúrgica es el tratamiento de elección, siendo curativa en la mayoría de los casos sin requerir terapia adyuvante. No se ha descrito potencial metastático $\mathrm{y}$, aunque infrecuente, es posible la recurrencia tras la resección. 4

\section{Caso Clínico}

Exponemos el caso de un paciente varón de 34 años, con antecedentes de esquizofrenia, que acude a nuestro servicio por presentar una tumoración dolorosa, de consistencia blanda, adherida a planos profundos y sin signos inflamatorios locales en eminencia tenar derecha de 13 años de evolución. No se constataron alteraciones motoras ni sensitivas.

El estudio radiográfico no mostró hallazgos significativos. Tras realizarse RNM con y sin gadolinio (-Figs. 1 y 2), se identifica una masa de características expansivas bien delimitada, con dos componentes comunicados: uno intermuscular heterogéneo de $4 \times 3 \mathrm{~cm}$ y otro superficial multilobulado que se desplaza hacia el túnel carpiano sin afectar al retináculo ni a los tendones flexores, y con un componente vascular importante que sugiere un hemangioma cavernoso con proliferación lipomatosa.

Ante nuestra sospecha diagnóstica, el largo tiempo de evolución de la lesión y la ausencia de signos de malignidad, se decide realizar extirpación del tumor sin toma de biopsia previa.

El paciente es intervenido quirúrgicamente bajo anestesia plexual e isquemia braquial, evidenciándose una tumoración diseminada por toda la palma de la mano, multilobulada, que se introduce en planos submusculares (-Fig. 3) y en la que se distinguen una masa de mayor tamaño con áreas de hematoma en su interior y otra de menor entidad compuesta por material de aspecto grumoso ( - Fig. 4), adherido ese último, a las ramas sensitivas del nervio mediano para el primer y segundo dedos (nervios digital palmar común del primer dedo y digital radial propio del segundo dedo respectivamente). Se realiza apertura del canal carpiano y microdisección con gafas

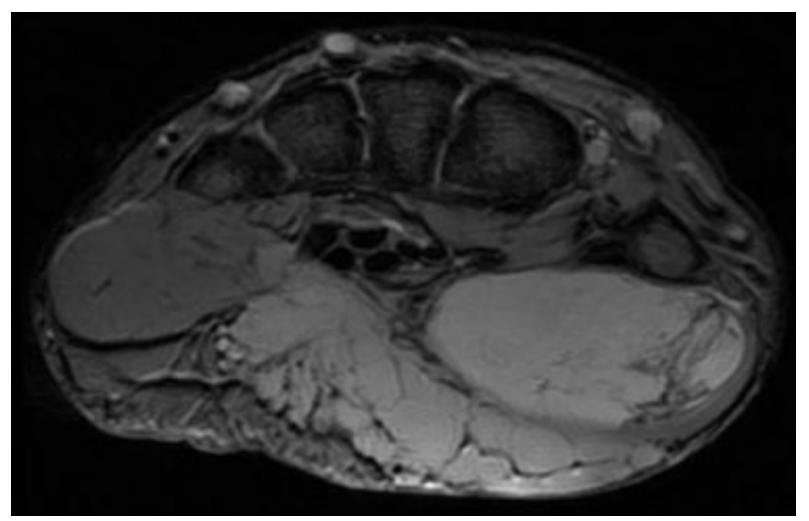

Fig. 1 Imagen de RNM sin contraste en secuencia T2 donde se muestra la hiperintensidad de la lesión así como la diferenciación de sus dos componentes: el intermuscular y el superficial multilobulado.

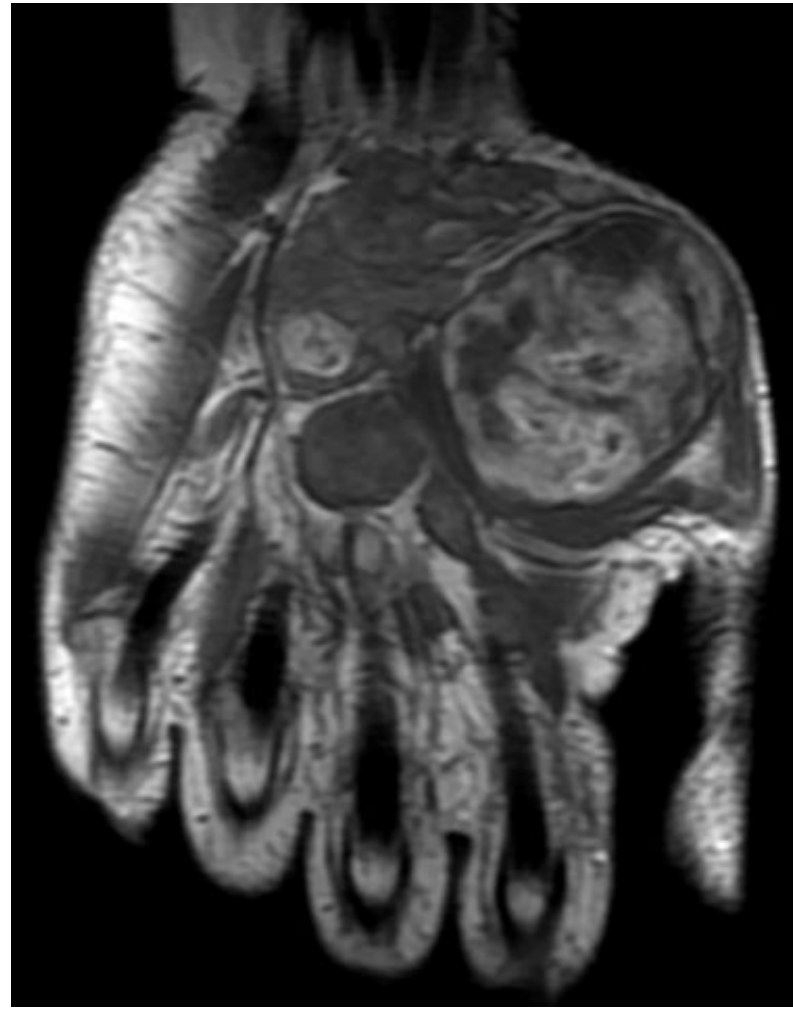

Fig. 2 En la RNM con contraste se observa la captación de gadolinio por la tumoración y el componente vascular de la misma.

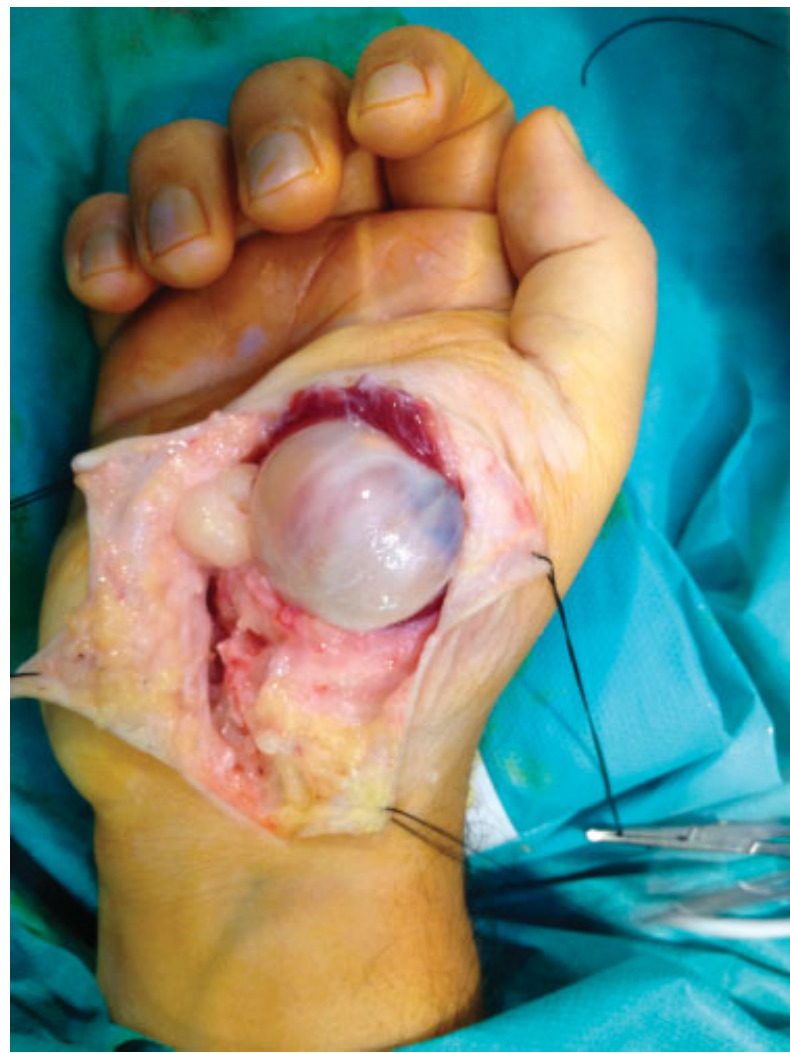

Fig. 3 Imagen intraoperatoria del tumor. Se observa la tumoración principal inmersa en el plano muscular. 


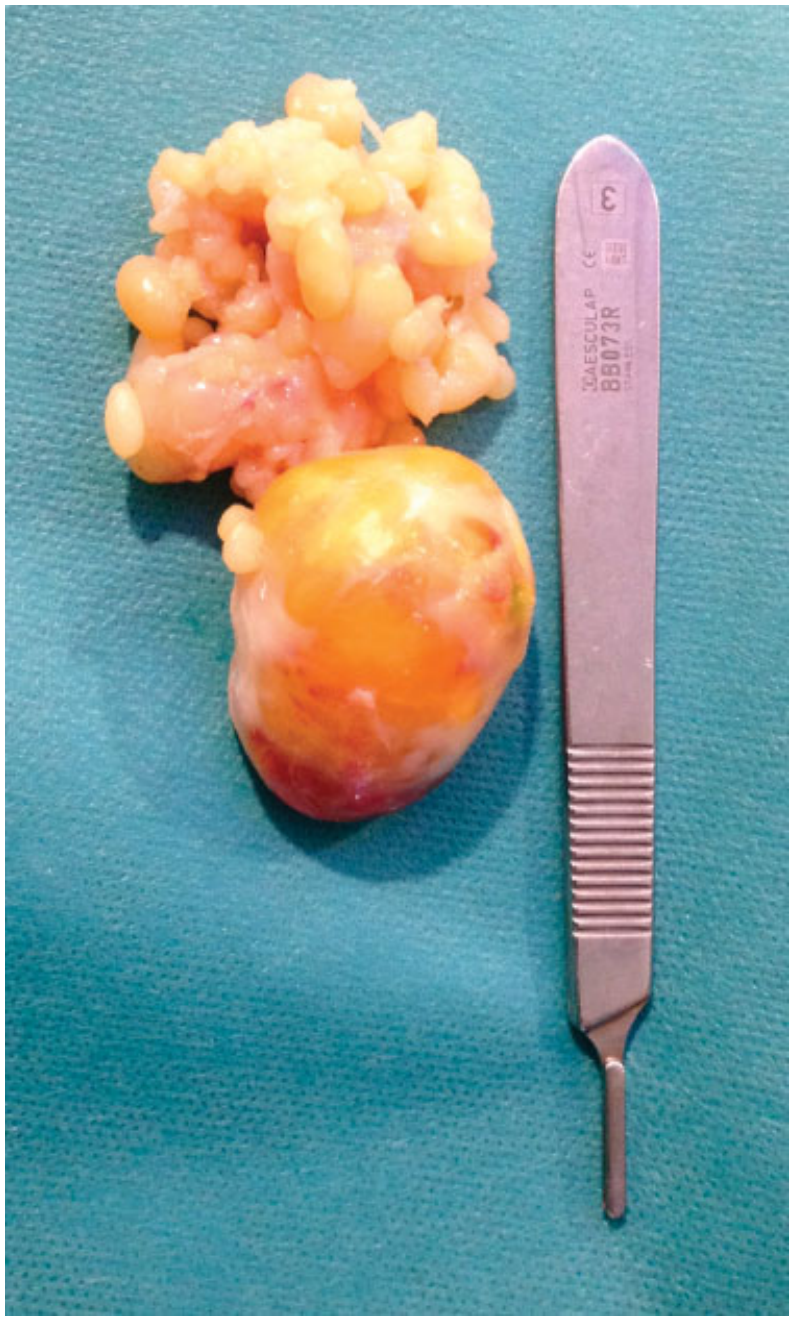

Fig. 4 Visión macroscópica de la lesión en la que se puede apreciar el componente lipomatoso dependiente de la masa nodular mayor.

lupa de x2.5 separando la tumoración de las fibras nerviosas del tronco del nervio mediano hasta su resección, extirpando el nervio digital palmar común del primer dedo y dejando un pequeño nódulo de aspecto lipomatoso difícilmente disecable del nervio digital radial propio para el segundo dedo.

En el estudio anatomopatológico, se identifican varios fragmentos de tejido fibroso de coloración blanquecinaamarillenta, uno de los cuales es una formación nodular de $4 \times 3 \times 2$ centímetros de superficie lisa, que a los cortes seriados presenta áreas de aspecto hemorrágico. Tras ser sometida a pruebas inmunohistoquímicas, se diagnostica de schwannoma plexiforme ( - Figs. 5 y 6 ).

El paciente es revisado cada tres meses, con una evolución favorable desde el postoperatorio. Tras once meses de la cirugía, se encuentra subjetivamente bien, realizando vida normal. Presenta atrofia de la eminencia tenar e hipostesia en primer dedo, sin limitación para la flexoextensión de los dedos ni la oposición del pulgar (-Figs. 7 y 8). Tampoco se han observado otras lesiones que puedan sugerir una asociación sindrómica.

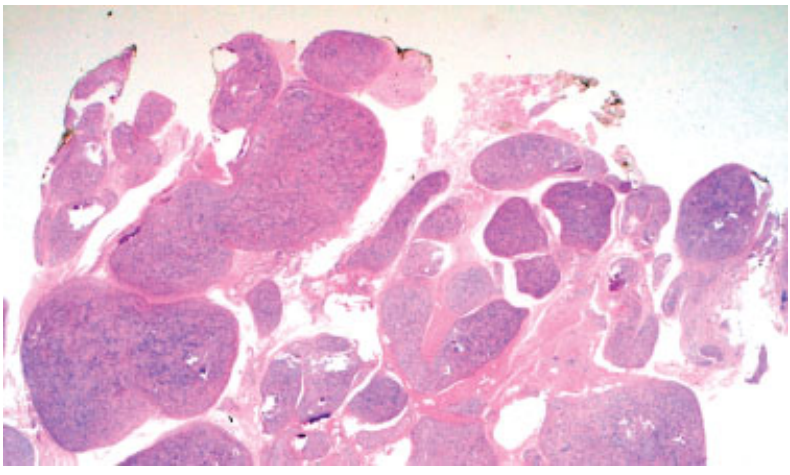

Fig. 5 Visión con lupa de los múltiples nódulos independientes característicos de este tumor.

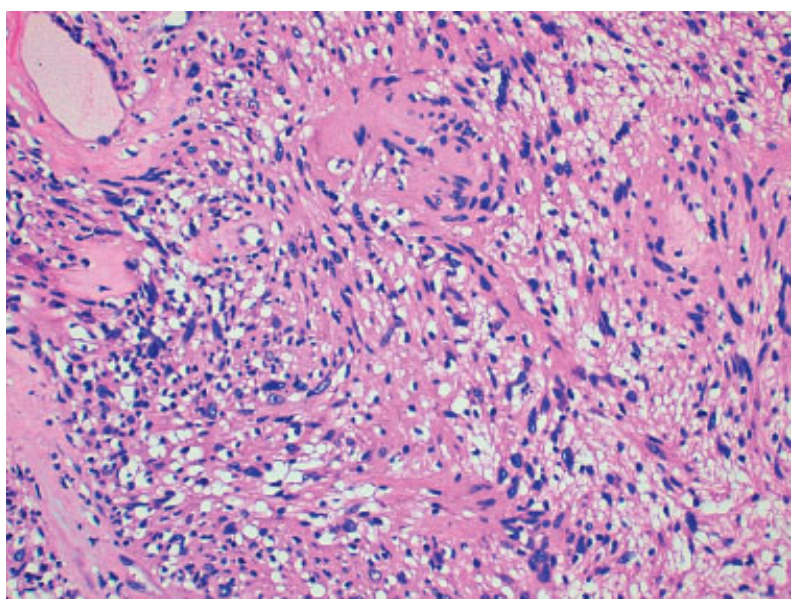

Fig. 6 Detalle de los núcleos en empalizada rodeando áreas de colágeno (hematoxilina-eosina, x20).

\section{Discusión}

Los schwannomas son los tumores benignos de estirpe nerviosa más frecuentes. De ellos, sólo un 1\% ocurren en las regiones de la muñeca y la mano, ${ }^{5}$ afectando principalmente a los nervios mediano y cubital. ${ }^{6}$

En su forma clásica, el schwannoma es fácilmente reconocible; sin embargo, han sido descritas algunas variantes que pueden causar dificultades diagnósticas. ${ }^{1}$

El schwannoma plexiforme es una variante rara de schwanoma caracterizada por su crecimiento intraneural, plexiforme y a menudo multinodular, compuesta únicamente por células de Schwann. ${ }^{2}$ Desde su primera descripción por Harkin y col., hasta la actualidad, alrededor de 100 casos han sido descritos en la literatura. ${ }^{2}$

En contraste con el schwannoma convencional, más frecuente en zonas flexoras de los miembros, el tronco, cabeza y cuello son las regiones de asiento más comunes para esa variante plexiforme. Suele aparecer en adolescentes y adultos jóvenes, como una masa única de crecimiento lento, bien circunscrita, móvil, a veces dolorosa, con un tamaño comprendido entre 0,5 y $2,5 \mathrm{~cm}$ de diámetro. ${ }^{3}$

Histológicamente se caracteriza por mostrar múltiples nódulos independientes, compuestos por una proliferación de células fusiformes conocidas como áreas de Antoni A con 


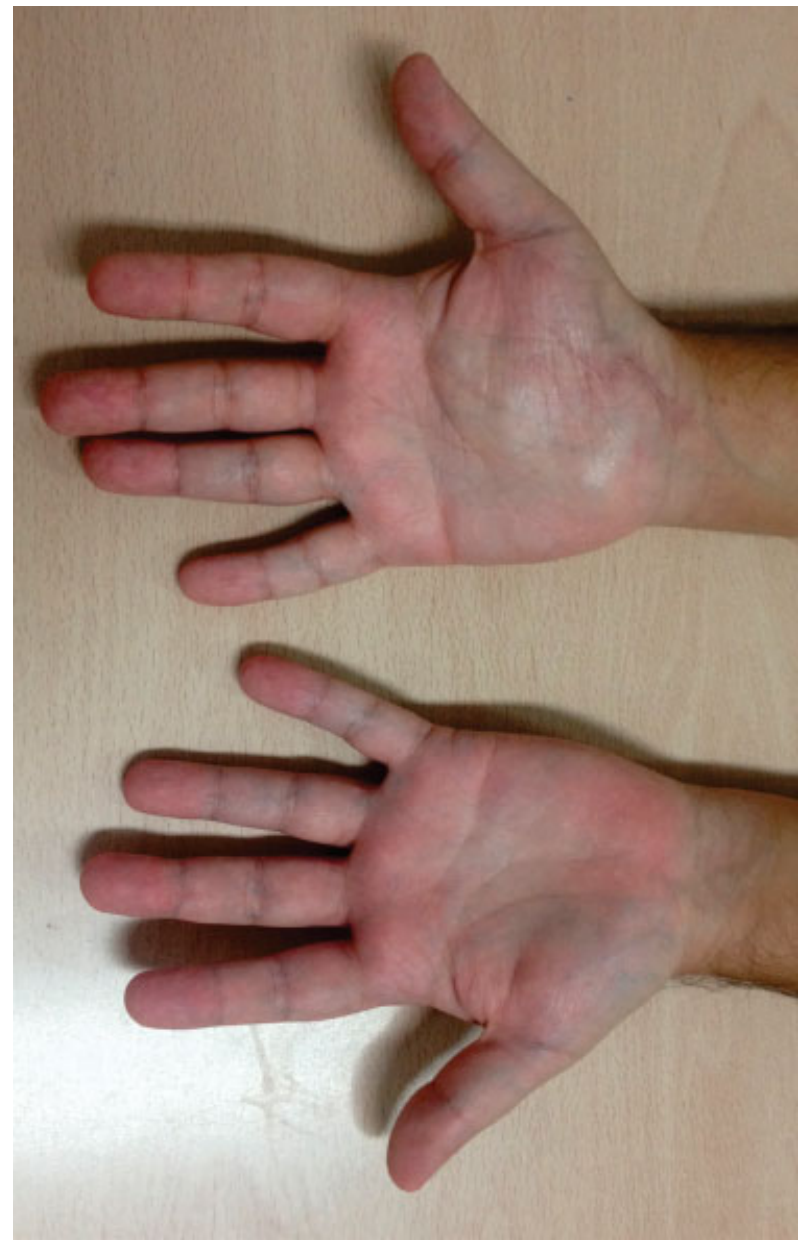

Fig. 7 Atrofia de la eminencia tenar apreciable tras 12 meses de la intervención.

núcleos que se disponen en empalizada formando los cuerpos de Verocay. $^{3}$ Las áreas Antoni B (zonas hipocelulares) son escasas en el schwannoma plexiforme. ${ }^{1}$

En nuestro caso esas características histológicas fueron observadas tanto en la masa nodular principal como en la de aspecto lipomatoso, aunque no podemos afirmar tras revisar

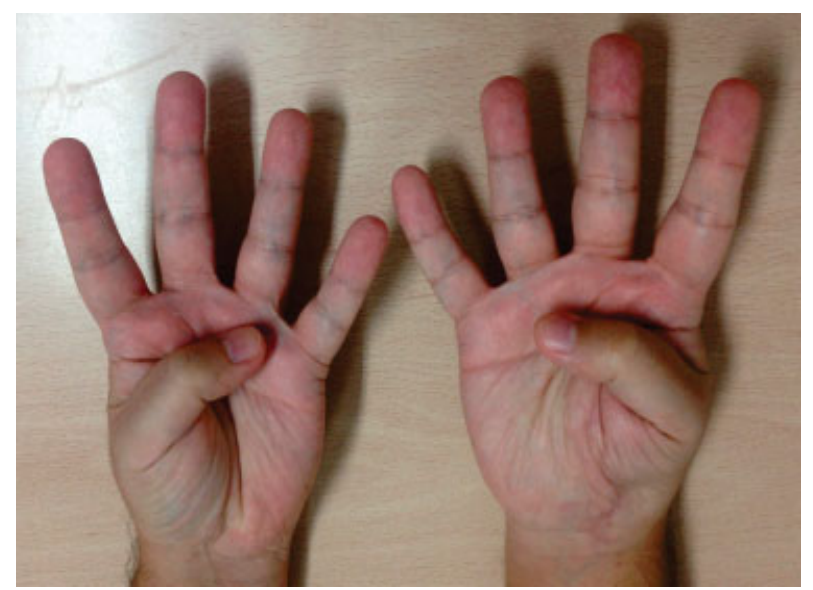

Fig. 8 El paciente no muestra limitación para la flexoextensión de los dedos ni la oposición del pulgar. la literatura que dicho bimorfismo macroscópico sea propio de este tumor.

La mayoría, al igual que en nuestro paciente, se presenta de modo esporádico, aunque la aparición de múltiples lesiones puede sugerir una asociación sindrómica: el 5\% de los schwannomas plexiformes se asocian a neurofibromatosis tipo 2 y otro $5 \%$ a schwannomatosis. No se ha descrito asociación con neurofibromatosis tipo $1 .^{2-4}$

Es importante realizar el diagnóstico diferencial con los tumores malignos de la vaina de los nervios y en especial con el neurofibroma plexiforme, debido a las implicaciones clínicas y pronósticas. Éste último es patognomónico de la neurofibromatosis tipo 1 y posee capacidad de malignización hasta en el $10 \%$ de los casos. ${ }^{3,4}$ Otras consideraciones diagnósticas de menor relevancia incluyen el schwannoma convencional con modularidad intratumoral, el neuroma encapsulado en empalizada y el neuroma traumático. ${ }^{2}$

Para un diagnóstico de certeza es preciso el análisis anatomopatológico del tumor y su sometimiento a pruebas de inmunohistoquímica, mostrando postividad para la proteína S-100. ${ }^{7}$

Las pruebas de imagen pueden servir de ayuda, especialmente la RNM, donde se observará un aumento de señal en T2. Sin embargo, esa técnica no ha demostrado ofrecer información suplementaria a la obtenida con ecografía; en la mayoría de los casos, las imágenes observadas en neurofibromas o schwannomas no son útiles en el diagnóstico diferencial. ${ }^{8}$ En el caso que presentamos, la hipervascularización tumoral evidenciada por RNM llevó al diagnóstico de presunción de hemangioma cavernoso.

En cuanto a la realización de biopsia previa a la extirpación quirúrgica, no hemos hallado en la literatura evidencias de su indicación en ese tipo de tumores. En nuestro caso, el diagnóstico de sospecha unido a la ausencia de signos de malignidad, nos llevó a optar por el tratamiento quirúrgico de entrada, pues no se ha demostrado necesidad de biopsia para descartar un proceso maligno en casos de sospecha de hemangioma mediante RNM. ${ }^{9}$

Aunque algunos autores defienden el tratamiento conservador reservando la cirugía para casos sintomáticos, ${ }^{7}$ el tratamiento de elección es la resección quirúrgica. Los hallazgos intraoperatorios de esa lesión, difieren de los encontrados en el schwannoma convencional. En general, los fascículos nerviosos pequeños asociados a schwannoma convencional pueden ser sacrificados sin consecuencias, pues no suelen transmitir potenciales de acción. Por el contrario, en casos de tumores multinodulares y $/ 0$ multifasciculares como el schwannoma plexiforme, es difícil resecar completamente las lesiones sin causar un déficit neurológico. ${ }^{10}$ En nuestro caso, la diseminación del tumor impedía la resección completa sin sacrificio de fibras nerviosas. La hipoestesia referida por nuestro paciente en el primer dedo es debida a la resección en bloque del nervio digital palmar común del pulgar, dada la diseminación y adherencia a fibras nerviosas del tumor en ese territorio. Con el objeto de no desensibilizar por completo la primera comisura, optamos por preservar el nervio digital radial propio del segundo dedo a expensas de mantener un pequeño nódulo 
tumoral a dicho nivel. Consideramos que la atrofia tenar aparente tras la cirugía, pudiera haber estado presente previamente bien por causa compresiva o bien por desuso, habiéndose hecho evidente tras la exéresis del tumor. El paciente continúa manteniendo los movimientos de abducción y oposición del pulgar.

Sólo han sido documentados casos ocasionales de recurrencia local, y nunca se ha descrito capacidad de transformación maligna. ${ }^{1-4}$ Nuestro paciente no ha mostrado hasta la fecha signos de crecimiento del resto tumoral. En caso de progresión, y dada la naturaleza benigna de la lesión, nos plantearíamos la realización de una nueva resección si dicha progresión se acompaña de una nueva sintomatología.

Conflictos de interés

Ninguno.

\section{Bibliografía}

1 Punia RS, Dhingra N, Mohan H. Cutaneous plexiform schwannoma of the finger not associated with neurofibromatosis. Am J Clin Dermatol 2008;9(02):129-131
2 Berg JC, Scheithauer BW, Spinner RJ, Allen CM, Koutlas IG. Plexiform schwannoma: a clinicopathologic overview with emphasis on the head and neck region. Hum Pathol 2008;39(05):633-640

3 Ko JY, Kim JE, Kim YH, Ro YS. Cutaneous plexiform schwannomas in a patient with neurofibromatosis type 2. Ann Dermatol 2009; 21(04):402-405

4 Talwalkar SC, Cutler L, Stilwell JH. Multiple plexiform schwannoma of the hand and forearm: a long-term follow-up. J Hand Surg Am 2005;30B(04):358-360

5 Gosk J, Gutkowska O, Urban M, Martynkiewicz J, Bąk M, Ziółkowski P. Benign nerve tumours of the hand (excluding wrist). Arch Orthop Trauma Surg 2015;135(12):1763-1769

6 Kütahya H, Güleç A, Güzel Y, Kacira B, Toker S. Schwannoma of the median nerve at the wrist and palmar regions of the hand: a rare case report. Case Rep Orthop 2013;2013:950106

7 Wollina U, Langner D, Gruner M, Schönlebe J, Haroske G. Isolated plexiform schwannoma of the hand. J Dermatol Case Rep 2008;2 (02):28-30

8 Amoretti N, Grimaud A, Hovorka E, Chevallier P, Roux C, Bruneton JN. Peripheral neurogenic tumors: is the use of different types of imaging diagnostically useful? Clin Imaging 2006;30(03):201-205

9 Wierzbicki JM, Henderson JH, Scarborough MT, Bush CH, Reith JD, Clugston JR. Intramuscular hemangiomas. Sports Health 2013;5 (05):448-454

10 Lim CY, Low TH, Sivanoli R, Teh KK, Thuraisingham R. Multinodular/plexiform schwannoma of the ulnar nerve. ANZ J Surg 2014;84(1-2):93-94 